\title{
ALTERNATING-PANCYCLISM IN 2-EDGE-COLORED GRAPHS $^{1}$
}

\author{
NARDA CoRDERO-Michel
}

AND

\section{Hortensia Galeana-SÁnChez}

Instituto de Matemáticas, Universidad Nacional Autónoma de México Ciudad Universitaria, México, D.F., C.P. 04510, México

e-mail: narda@matem.unam.mx hgaleana@matem.unam.mx

\begin{abstract}
An alternating cycle in a 2-edge-colored graph is a cycle such that any two consecutive edges have different colors. Let $G_{1}, \ldots, G_{k}$ be a collection of pairwise vertex disjoint 2-edge-colored graphs. The colored generalized sum of $G_{1}, \ldots, G_{k}$, denoted by $\oplus_{i=1}^{k} G_{i}$, is the set of all 2-edge-colored graphs $G$ such that: (i) $V(G)=\bigcup_{i=1}^{k} V\left(G_{i}\right)$, (ii) $G\left\langle V\left(G_{i}\right)\right\rangle \cong G_{i}$ for $i=1, \ldots, k$ where $G\left\langle V\left(G_{i}\right)\right\rangle$ has the same coloring as $G_{i}$ and (iii) between each pair of vertices in different summands of $G$ there is exactly one edge, with an arbitrary but fixed color. A graph $G$ in $\oplus_{i=1}^{k} G_{i}$ will be called a colored generalized sum (c.g.s.) and we will say that $e \in E(G)$ is an exterior edge if and only if $e \in E(G) \backslash\left(\bigcup_{i=1}^{k} E\left(G_{i}\right)\right)$. The set of exterior edges will be denoted by $E_{\oplus}$. A 2-edge-colored graph $G$ of order $2 n$ is said to be an alternating-pancyclic graph, whenever for each $l \in\{2, \ldots, n\}$, there exists an alternating cycle of length $2 l$ in $G$.

The topics of pancyclism and vertex-pancyclism are deeply and widely studied by several authors. The existence of alternating cycles in 2-edgecolored graphs has been studied because of its many applications. In this paper, we give sufficient conditions for a graph $G \in \oplus_{i=1}^{k} G_{i}$ to be an alternating-pancyclic graph.
\end{abstract}

Keywords: 2-edge-colored graph, alternating cycle, alternating-pancyclic graph.

2010 Mathematics Subject Classification: 05C38.

\footnotetext{
${ }^{1}$ This research was supported by grants UNAM-DGAPA-PAPIIT IN102320.
} 


\section{REFERENCES}

[1] J. Bang-Jensen and G. Gutin, Alternating cycles and paths in edge-coloured multigraphs: A survey, Discrete Math. 165/166 (1997) 39-60. doi:10.1016/S0012-365X(96)00160-4

[2] J. Bang-Jensen and G. Gutin, Alternating cycles and trails in 2-edge-coloured complete multigraphs, Discrete Math. 188 (1998) 61-72. doi:10.1016/S0012-365X(97)00274-4

[3] J. Bang-Jensen and G.Z. Gutin, Digraphs: Theory, Algorithms and Applications (Springer-Verlag London, Ltd, London, 2009). doi:10.1007/978-1-84800-998-1

[4] W.S. Chou, Y. Manoussakis, O. Megalakaki, M. Spyratos and Zs. Tuza, Paths through fixed vertices in edge-colored graphs, Math. Sci. Hum. 127 (1994) 49-58.

[5] A. Contreras-Balbuena, H. Galeana-Sánchez and I.A. Goldfeder, A new sufficient condition for the existence of alternating Hamiltonian cycles in 2-edge-colored multigraphs, Discrete Appl. Math. 229 (2017) 55-63. doi:10.1016/j.dam.2017.04.033

[6] N. Cordero-Michel and H. Galeana-Sánchez, Vertex alternating-pancyclism in 2 edge-colored generalized sum of graphs, Discrete Appl. Math 284 (2020) 281-289. doi:10.1016/j.dam.2020.03.047

[7] P. Das, Pan-alternating cyclic edge-partitioned graphs, Ars Combin. 14 (1982) $105-114$.

[8] D. Dorninger, On permutations of chromosomes, in: Contributions to General Algebra 5, Proceedings of the Salzburg Conference, May 29-June 1, 1986, HölderPichler-Tempsky (Ed(s)), (Teubner-Verlag, Stuttgart, 1987) 95-103.

[9] D. Dorninger, Hamiltonian circuits determining the order of chromosomes, Discrete Appl. Math. 50 (1994) 159-168. doi:10.1016/0166-218X(92)00171-H

[10] D. Dorninger and W. Timischl, Geometrical constraints on Bennett's predictions of chromosome order, Heredity 59 (1987) 321-325. doi:10.1038/hdy.1987.138

[11] S. Fujita and C. Magnant, Properly colored paths and cycles, Discrete Appl. Math. 159 (2011) 1391-1397. doi:10.1016/j.dam.2011.06.005

[12] A. Gorbenko and V. Popov, The Hamiltonian alternating path problem, IAENG Int. J. Appl. Math. 42 (2012) 204-213.

[13] L. Gourves, A. Lyra, C. Martinhon and J. Monnot, The minimum reload $s-t$ path, trail and walk problems, Discrete Appl. Math. 158 (2010) 1404-1417. doi:10.1016/j.dam.2010.03.009

[14] R. Häggkvist and Y. Manoussakis, Cycles and paths in bipartite tournaments with spanning configurations, Combinatorica 9 (1989) 33-38. doi:10.1007/BF02122681 
[15] J. Petersen, Die Theorie der regulären graphs, Acta Math. 15 (1891) 193-220. doi:10.1007/BF02392606

[16] P.A. Pevzner, DNA physical mapping and alternating Eulerian cycles in colored graphs, Algorithmica 13 (1995) 77-105. doi:10.1007/BF01188582

[17] G. Wang and H. Li, Color degree and alternating cycles in edge-colored graphs, Discrete Math. 309 (2009) 4349-4354. doi:10.1016/j.disc.2009.01.016

[18] H.C. Wirth and J. Steffan, Reload cost problems: minimum diameter spanning tree, Discrete Appl. Math. 113 (2001) 73-85. doi:10.1016/S0166-218X(00)00392-9

[19] H. Xu, D.M. Kilgour, K.W. Hipel and G. Kemkes, Using matrices to link conflict evolution and resolution in a graph model, European J. Oper. Res. 207 (2010) 318-329. doi:10.1016/j.ejor.2010.03.025

[20] H. Xu, K.W. Li, K.W. Hipel and D.M. Kilgour, A matrix approach to status quo analysis in the graph model for conflict resolution, Appl. Math. Comput. 212 (2009) 470-480. doi:10.1016/j.amc.2009.02.051

[21] H. Xu, K.W. Li, D.M. Kilgour and K.W. Hipel, A matrix-based approach to searching colored paths in a weighted colored multidigraph, Appl. Math. Comput. 215 (2009) 353-366.

doi:10.1016/j.amc.2009.04.086

Received 26 October 2019

Revised 14 July 2020

Accepted 16 July 2020 